
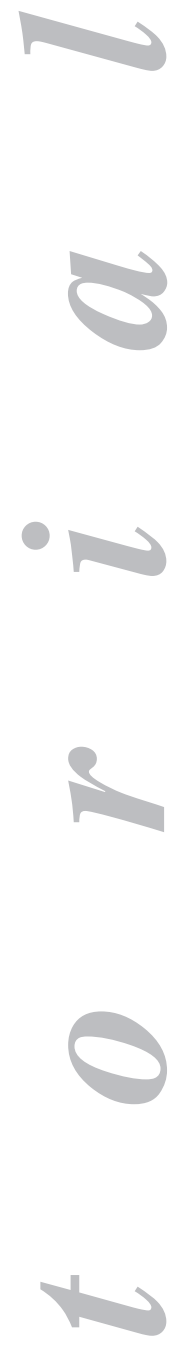

0
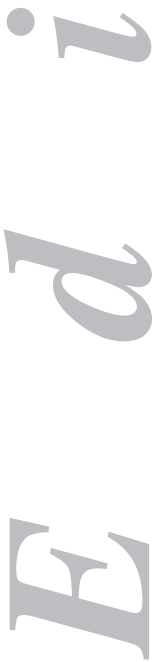

\section{Increasing the detection of hepatotoxic response in clinical practice: a quality commitment}

Modern drug therapy came of age in the early 19th century, when it abandoned the secondary role it had been relegated to by the more prestigious diagnostic process during the past few decades. The outstanding advances occurred in both cellular and molecular biology, as well as in biotechnology, had a lot to do with this, since they -together with refined diagnostic tests- favored the development of new biologic drugs, and an unprecedented growth of clinical research with the aim of providing a rigorous scientific assessment of therapeutic benefits and drug-related toxicity.

However, a point that has drawn greater attention during the last few years is the safety of patients exposed to drugs. In fact, many drugs exhibit dazzling efficacy but on occasion become double-edged swords, and such safety issues pose a true challenge not only to the scientific community, but to society itself. The fast spread of drugs within a global market determines large-scale exposure of patients to new molecules, with a potential for greater numbers of unexpected adverse effects. The continual commercialization of new drugs, the increasing number of drugs used by the population, and the increasing utilization of pharmacologically active "alternative medications" are all the hallmark of current society and favor the likely development of adverse events.

Recent studies contributed to bring to the fore the significance of drug-related adverse events. In 1998 Lazarou et al. (1) reported that their estimated incidence of serious adverse events among inpatients in the U.S. was 6.7\% ( 1 in every 15 inpatients), while adverse events with a fatal outcome amounted to $0.32 \%$ and thus involved a total of 106,000 patients, which represents the $4^{\text {th }}$ to $6^{\text {th }}$ leading cause of mortality. Even when considered exaggerated, nobody would argue that these figures deserve both attention and active policies by the health systems. Indeed, the true risks of medications only manifest when drugs leave the well-controlled "clinical trial research" setting -where populations at lower risk are commonly examined, the presence of underlying diseases and the concurrent use of other drugs represent exclusion criteria, and exposure length is relatively short- and move on to be administered within a "naturalistic" context, namely standard clinical practice. These considerations are supported by a recent study in the U.S., where drugs that would be eventually withdrawn from the market on safety grounds had already been administered to 20 million subjects (2). The fact that almost $10 \%$ of the adult population had already been exposed to non-essential drugs before their harmful effects were properly identified is strong evidence that new systems for the early recogni-

This paper was partly supported by grant FISS 02/1167. 
tion of medication-related toxicity risks need to be implemented. Along these lines, a clear example is the recent withdrawal of the lipid-lowering agent cerivastatin, which has had a huge impact on the media, and put in the limelight the great relevance post-marketing drug surveillance has to the actual goal of drug therapy benefits with acceptable risks.

Not surprisingly the liver is also a target organ for adverse reactions, as it plays a key role in the process of drug metabolism. Since such potential adverse event is of extreme severity, it has become a major cause of drug withdrawal from the pharmaceutical market or of restricted indications (3). Examples of drugs withdrawn from the market during the past decade include ebrotidine, tolcapone, nefazodone, tetrabamate, nimesulide, and trovafloxacin.

Idiosyncratic adverse reactions of the liver during drug therapy are uncommon events with a rate of $1 / 10,000$ to $1 / 100,000$ exposures. Sensitive methods to detect idiosyncratic hepatotoxins exerting their effects through metabolic or immunoallergic mechanisms are still unavailable during preclinical development. Once the drug is on the market, hepatotoxicity-related events are basically diagnosed by ruling out alternative origins in the absence of specific markers of liver injury of toxic origin. Thus, it is hardly surprising that hepatotoxicity is rarely recognized and many events go unnoticed. It is estimated, however, that liver disease of toxic origin represents $1 / 600$ to $1 / 3500$ of all hospital admissions, roughly $2-5 \%$ of jaundice cases in inpatients, and $10 \%$ of hepatitis cases (4), with the most common presentation of hepatic adverse reactions being a syndrome similar to acute hepatitis. A prospective, multicenter study by the Acute Liver Failure Study Group (5) on the etiology of acute liver failure showed that toxicity currently heads the list of all potential causes when cases associated with acetaminophen toxicity (39\%) and idiosyncratic reactions $(13 \%)$ are added together. Nonetheless, a significant proportion of serious acute liver failure cases remain to be explained following thorough diagnostic evaluation. A recent study (6) in patients with serious idiopathic acute liver failure revealed the presence of acetaminophen-protein adducts (a surrogate marker of acetaminophen-related liver injury) in the serum of $30 \%$ of cases, which shed new evidence on the responsibility of drugs for at least one part of serious acute liver failure cases of unknown origin.

The practical usefulness of establishing costly, complex surveillance systems for the earliest detection of adverse reactions that -as is the case with hepatotoxic events- are mostly unpredictable may be challenged. However, three reasons at least could be summoned to support the case for early detection strategies. First, the most determining factor in the severity and possibly fulminant outcome of a hepatotoxic reaction of hepatocellular type has been seen to be treatment continuation with the responsible agent once symptoms become apparent (7). Secondly, the identification of relevant hepatotoxicity cases may allow the establishment of risk-benefit profiles for involved drugs, as well as the finding of toxicity risk and individual susceptibility factors to help take measures in preventing new cases. Finally, there is a practical reason for the patient, namely the prevention of new events that might be more serious than the index episode upon re-exposure to the responsible drug.

There is nonetheless an almost complete absence of epidemiologic studies designed to prospectively investigate the relationship between drug agents and liver disease. Their limitations mainly stem from data sources, their definition of liver injury, and inherent difficulties to establish a causality link in the setting of polymedicated patients. In Spain a Spontaneous Reporting Program for Adverse Reactions, dubbed the yellow card, is in existence since 1983, and represents the essential core 
of the Spanish Drug Surveillance System. This post-marketing passive surveillance program has allowed warning signs of new risks to be generated, a characterization of drug-induced syndromes, and a comparison of toxicity profiles for drugs within given groups, but underreporting is possibly its most significant limitation despite obligatoriness (8). At best, the reporting of serious adverse events -and hepatotoxicity is such an event- has been estimated in 1 to $10 \%$ of actual figures. The lower the reporting of events, the longer the delay in the detection of drug safety problems. This system's limitations also include difficulties in obtaining reliable information on the nature and time sequence of events; in the absence of a well-defined population source, the denominator or exposed population is often unknown. The latter limitation is similarly shared by both case and series reports, which also allow hypothesis formulation and usually focus on exceptional cases or high-risk populations.

The real rate of drug-related cases of liver disease is unknown. The only prospective, population-based incidence study performed in France found an annual crude incidence rate of $13.9 \pm 2.4$ per 100,000 population, which represents an incidence of hepatotoxicity 16 times higher than that reported to regulatory authorities (9). Incidence figures for hepatotoxicity obtained from a Hepatotoxicity Registry Coordinating Center in operation from 1994 were $34.2 \pm 10.7$ cases per $10^{6}$ population per year for the period 1998-2003, with approximately half of these requiring hospital admission (10). Although lower when compared to those in the French study (9), these figures remain outstanding and higher than those reported by a recent study in Catalonia (11).

Along this line, the paper by Ruiz-Montero et al. (12) that is being published in this issue of Revista Española de Enfermedades Digestivas discusses an active strategy to increase the detection of toxic liver disease in inpatients using a number of target departments to bring the number of identified reactions nearer the actual incidence. The study's methodology was designed to sensitize healthcare professionals by putting them on permanent alert as the most effective means of increasing the number of hepatotoxicity cases reported. In applying this procedure the authors obtained an estimated incidence of liver toxicity of 6.74 cases per 100,000 populations in their setting. Even considering this rate an underestimation of the actual incidence of liver toxicity in the authors' area of influence, which would result from the study's design not including all hospital departments, it still represents an incidence four-fold higher than that reported to health authorities during the same period. Another issue deserving comment is the added value brought to achieving this goal by the Clinical Pharmacology Department. Their acting coordinately with hepatologists and related specialists in each area, where they brought to bear their characteristic pharmacoepidemiologic methodology, has no doubt contributed to the training of clinicians and to increasing diagnostic suspicion; it has also facilitated the collection of detailed information for causality, and all of it without ever overloading the daily schedule of physicians, as the authors themselves literally quote.

A valid estimation of the incidence of an adverse event in a population should rely on a number of basic requirements, including the event's thorough documentation, a sensitive and specific screening method, and a clearly delimited population. Propective studies using automatic signals generated from laboratory data -a monitoring system that may be subsequently implemented on a permanent basis in hospitals- suggest that the development of adverse reactions in inpatients is a common fact. This procedure, which may be theoretically applicable to the identification of other laboratory changes such as drug-related agranulocytosis or bone marrow apla- 
sia, has been assessed in the detection of hepatotoxic adverse events and their relative proportion to other etiologies of liver disease in some studies, whose pooled analysis is made difficult by their differing definitions of liver damage. In a study carried out in one of the health areas in the United Kingdom it was found that ischemic hepatitis and pancreatobiliary disease represented $74 \%$ of cases when using the criterion of serum aspartate aminotransferase concentrations above $400 \mathrm{U} / \mathrm{L}$, whereas hepatotoxicity was responsible for $8.8 \%$ (13). These signals were misinterpreted or misdiagnosed in 52\% of cases. However, in a study carried out in France using as definition of liver damage a two-fold increase in serum alanine-aminotransferase activity identical figures were found for drug-related liver injury (14); in our setting (15), using internationally accepted criteria for liver injury (16), we found a prevalence of hepatotoxicity of $4 \%$. In the latter study, most common causes of liver biochemistry changes included pancreatobiliary, neoplastic, and ischemic disorders (15). A limitation of this type of design is its uncertain clinical relevance, unless robust parameters for liver injury (e. g., serum alanine-aminotransferase concentrations ranging from 5 to 10 times the upper limit of normality; or even better serum bilirubin levels on the verge of clinical expression, i. e., $3 \mathrm{mg} / \mathrm{dL}$ ) are selected. Otherwise, patients with minor drug-related or -unrelated changes in liver profile may be detected, and many of the latter subjects even "adjust" in spite of therapy continuation and never develop overt liver toxicity, as has been shown with some drugs (statins, isoniazide).

In view of this information we may reflect on the poor performance of this methodology to detect hepatotoxic adverse reactions because of their actual frequency; however, the benefits inherent to the training of clinicians' sensitivity cannot be overemphasized, since these alterations often go unnoticed or misclassified by physicians as previously mentioned.

In short, any initiatives to promote "pharmacoepidemiological culture" in healthcare providers should be welcomed. The fact that the diagnosis of hepatotoxicity, in contrast to other diseases, has not benefited from the extraordinary advances in genomics, proteomics, and radiodiagnosis as yet, and still gravitates around careful history taking and a correct interpretation of the patient's clinical manifestations and laboratory data, should not be forgotten. The ultimate objective of any strategy to detect and gain insight into hepatotoxic adverse events may still be summarized in the old hippocratic aphorism "primun non nocere".

M. I. Lucena and R. J. Andrade ${ }^{1}$

Service Clinical Pharmacology. ${ }^{1}$ Unit of Hepatology. Service of Digestive Diseases. Hospital Universitario Virgen de la Victoria. Faculty of Medicine. Málaga, Spain

\section{References}

1. Lazarou J, Pomeranz BH, Corey PN. Incidente of adverse drug reactions in hospitalizad patients: a metaanalysis of prospective studies. JAMA 1998; 279: 1200-5.

2. Friedman MA, Woodcock J, Lumpkin MM, Shuren JE, Hass AE, Thompson LJ. The safety of newly approved medicines: do recent market removals mean there is a problem? JAMA 1999; 281: 1728-34.

3. Arnaiz JA, Carne X, Riba N, Codina C, Ribas J, Trilla A. The use of evidence in pharmacovigilance. Case reports as the reference source for drug withdrawals. Eur J Clin Pharmacol 2001; 57: 89-91.

4. Døssing, M, Sonne J. Drug-induced hepatic disorders. Incidence, management and avoidance. Drug Safety 1993; 9: 441-9. 
5. Ostapowicz G, Fontana RJ, Schiodt FV, et al. Results of a prospective study of acute liver failure at 17 Tertiary Care centers in the United States. Ann Intern Med 2002; 137: 947-57.

6. Davern TJ, James L, Larson A, et al. Serum acetaminophen adducts identify patients with severe aceta-minophen toxicity [abstract]. Hepatology 2003; 34: 539A.

7. Zimmmerman HJ. Hepatotoxicity: the adverse effects of drugs and other chemicals on the liver. Philadelphia: Lippincott Williams \& Wilkins, 1999.

8. Grupo IFAS (Industria Farmacéutica y Administración Sanitaria). Nuevas perspectivas de la farmacovigilancia en España y en la Unión Europea. Madrid: Jarpyo Editores, 1998.

9. Sgro C, Clinard F, Ouazir K, Chanay H, Allard C, Guilleminet C, et al. Incidence of drug-induced hepatic injuries: a French population-based study. Hepatology 2002; 36: 451-5.

10. Andrade RJ, Lucena MI, Fernández MC, et al. Toxic and drug-induced liver injury: an analysis of 461 incidences submitted to a Registry over a 10-year period. Gastroenterology, 2005.

11. Ibáñez L, Pérez E, Vidal X, Laporte JR, the Grup d'Estudi Multicentric d'Hepatotoxicitat Aguda de Barcelona (GEMHAB). Prospective surveillance of acute serious liver disease unrelated to infectious, obstructive, or metabolic diseases: epidemiological and clinical features, and exposure to drugs. J Hepatol 2002; 37: 592600.

12. Ruiz-Montero A, Durán Quintana JA, Jiménez Sáenz M, Abadín Delgado JA. Una estrategia para mejorar la detección de hepatotoxicidad por medicamentos. Rev Esp Enferm Dig 2005; 97: 155-60.

13. Whitehead MW, Hawkes ND, Hainsworth I, Kingham JGC. A prospective study of the causes of notably raised aspartate aminotransferase of liver origin. Gut 1999; 45: 12933.

14. Bagheri H, Michel F, Lapeyre-Mestre M, Lagiere E, Cambus JP, Valdiguie P, et al. Detection and incidence of drug-induced liver injuries in hospital: prospective analysis from laboratory signals. Br J Clin Pharmacol 2000; 50: 479-84.

15. Benichou C. Report of an International Consensus Meeting. Criteria of drug-induced liver disorders. J Hepatol 1990; 11: 272-6.

16. Fernández EM, Verge C, Puche M, Morales M, Lucena M, Andrade R, et al. Identificación precoz de hepatotoxicidad mediante la utilización de señales de alerta bioquimica en un hospital de tercer nivel: Estudio Preliminar. Rev Esp Enferm Dig 2003; 95 (Supl. I): 120. 